\title{
BMJ Open Creating dementia-friendly and inclusive communities for social inclusion: a scoping review protocol
}

\author{
Lillian Hung (D) , ${ }^{1,2}$ Sharon Leitch, ${ }^{3}$ Ryan Hung, ${ }^{4}$ Alison Phinney ${ }^{5}$
}

To cite: Hung L, Leitch S, Hung $\mathrm{R}$, et al. Creating dementia-friendly and inclusive communities for social inclusion: a scoping review protocol. BMJ Open 2020;10:e035028. doi:10.1136/ bmjopen-2019-035028

- Prepublication history and additional material for this paper are available online. To view these files, please visit the journal online (http://dx.doi. org/10.1136/bmjopen-2019035028).

Received 16 0ctober 2019 Revised 29 March 2020 Accepted 21 May 2020

Check for updates

(C) Author(s) (or their employer(s)) 2020. Re-use permitted under CC BY-NC. No commercial re-use. See rights and permissions. Published by BMJ.

${ }^{1}$ Gerontology, Simon Fraser University, Vancouver, British Columbia, Canada

${ }^{2}$ Nursing, Vancouver General Hospital, Vancouver, British Columbia, Canada

${ }^{3}$ Business, Kwantlen Polytechnic University, Surrey, British

Columbia, Canada

${ }^{4}$ Science, Simon Fraser University, Burnaby, British Columbia, Canada

${ }^{5}$ Nursing, University of British Columbia, Vancouver, British Columbia, Canada

Correspondence to

Dr Lillian Hung;

lillian.hung@vch.ca

\section{ABSTRACT}

Introduction The number of people with dementia is increasing worldwide, with the majority of people with dementia living at home in the community. WHO calls for global action on the public health response to dementia. Social exclusion is commonly reported by people with dementia and their families. Dementia-friendly and inclusive community has emerged as an idea that holds potential to contribute to the mitigation of social exclusion. The objective of the scoping review is to answer two questions: What social inclusion strategies that have been reported in the dementia-friendly and inclusive communities' literature? What strategies for developing dementia-friendly and inclusive communities that have shown to improve social inclusion?

Methods and analysis This scoping review will follow the Joanna Briggs Institute scoping review methodology and will take place between April and September 2020. The proposed review will consider studies based in community settings with participants living at home with early to late stages of dementia and their families. This includes a three-step search strategy: (1) to identify keywords from MEDLINE and CINAHL; (2) to conduct a second search using all identified keywords and index terms across selected databases (MEDLINE, CINAHL, AgeLine, PsycINF0, Web of Science, ProQuest and Google) and (3) to handsearch the reference lists of all included articles and reports for additional studies. Further, we will search Google for grey literature on published organisational reports. Two researchers will screen titles and abstracts independently and then assess the full text of selected citations against inclusion criteria. Extracted data will be presented in a narrative accompanied by tables that reflect the objective of the review.

Ethics and dissemination As the methodology of this study consists of collecting data from publicly available articles, it does not require ethics approval. This scoping review provides an overview of current evidence on strategies that support dementia-friendly and inclusive communities for social inclusion. The findings will offer insights to inform strategies for education, practice, policy and future research. We will share the scoping review results through conference presentations and an openaccess publication in a peer-reviewed journal.

\section{INTRODUCTION}

The number of people with dementia is growing exponentially across the world.

\section{Strengths and limitations of this study}

- People with lived experience, families of people with dementia, leaders of health organisations and community representatives will be involved in conducting and disseminating the scoping review.

- This is a novel and timely topic to understand how dementia-friendly and inclusive community may support social inclusion among people with dementia living at home in the community.

- The results and recommendations of scoping reviews cannot be graded since methodological appraisal of the quality of included studies is not consistent with the Joanna Briggs Institute scoping review guidelines.

- Studies not published in English will be missed.

- Strategies for building dementia friendliness in hospitals and other formal healthcare settings will not be captured.

Globally, around 50 million people live with dementia; there are nearly 10 million new cases every year. ${ }^{1}$ People with dementia and their informal caregivers face challenges that include stigma, social exclusion, difficulty accessing formal and informal support resources and financial pressures. WHO has declared dementia as a public health priority. ${ }^{1}$ The global action plan calls for actions to address dementia as a public health priority, increase awareness of dementia, establish dementia-friendly initiatives, provide support for dementia carers, and conduct research and innovation. Social exclusion and isolation can have detrimental effects on mental and physical health, such as depression and cardiovascular health. ${ }^{2}$ Current efforts to promote dementia-friendly and inclusive communities have the potential to promote social inclusion, change attitudes and behaviours, and support people with dementia to be engaged in community in meaningful ways. ${ }^{3}$

Dementia-friendly and inclusive community, social inclusion

A dementia-friendly and inclusive community can be defined as a city, town or village where 
people with dementia are understood, respected and supported. ${ }^{4}$ Social inclusion refers to a dynamic process where people engage with, and are part of, their social networks in the community to maintain meaningful social relations. ${ }^{5}$ Social connection and a sense of belonging are essential to well-being and quality of life. ${ }^{5}$ Purposeful connections, doing meaningful activities together, are important to a person with dementia and their families/ care providers. ${ }^{67}$ People with dementia can benefit from their local community network; social inclusion and social participation promote a sense of social citizenship, safety and contribution. ${ }^{8}$ The existing literature suggests that there is potential for dementia-friendly and inclusive community to support social inclusion. ${ }^{9}$ Stigma is one of the key challenges that people with dementia face. Stigma and lack of understanding cause people in societies to behave in ways that focus on problems of the disease rather than supporting the abilities that people with dementia have. ${ }^{10}$ Considering stigma and social exclusion are important issues for people with dementia living in the community, interventions that engage and include people with dementia in community activities seem vital to support people with dementia to remain living in their own homes for as long as possible.

The notion of dementia-friendly community has been drawn from the Age-Friendly Cities initiative of WHO. ${ }^{11}$ Age-friendly communities involve bringing stakeholders together to help create more inclusive environments in local communities in order to promote active and healthy ageing. ${ }^{12}$ Age-friendly communities contribute to good health and allow people to continue to participate fully in society. ${ }^{4}$ A similar guiding principle that dementia-friendly and age-friendly strategies both embody is-empowering local stakeholders to collaborate and contribute for social inclusion. Public education, reduction of stigma and removal of barriers in physical and social environments are common themes in dementia-friendly initiatives as well. ${ }^{13}$ Dementia advocates Rahman and Swaffer have criticised the term 'dementia-friendly' for its implied focus on deficit, suggesting a sense of otherness identified with an illness model, and thus, they argue for an assets-based focus. ${ }^{14}$ We acknowledge the need to shift the attention to strengths and assets that people with dementia can bring. Thus, we use the term 'dementia-friendly and inclusive' for the scoping review. While 'dementia-friendly' is the most common term used in the literature and government initiative, it is important to pay attention to language that promotes inclusion and human rights, not deficits.

In the UK, dementia-friendly and inclusive communities were found in regions with a large population of people living with dementia. ${ }^{15}$ Yet, people with dementia reported that they did not know how to contribute to their local community, and that they had an over-riding sense of low expectation and low self-belief. ${ }^{16}$ As the development of dementia-friendly and inclusive community has the potential to empower people with dementia, it is important to better understand what makes dementiafriendly and inclusive communities effective. ${ }^{17} 18 \mathrm{In}$
Australia, people with dementia identified six priorities in creating dementia-friendly communities: increased public awareness and understanding of dementia, access to social activities, support to stay at home, appropriate healthcare, transport and improvements to the physical environment. ${ }^{19}$ Stigma was viewed as a key barrier to community engagement, thereby leading to social isolation among people with dementia. ${ }^{20}$ In Canada, the notion of social inclusion has been underlined as essential for people with dementia. ${ }^{5}$ The impact of both supportive physical and social environments has been identified as vital to social inclusion, empowerment and enablement for people with dementia to contribute to and participate in society. ${ }^{21}{ }^{22}$ Active involvement of people with dementia and their families is also considered to be a key enabler in the development of dementia-friendly and inclusive community. ${ }^{23}$ 'Nothing about us without us' is a phrase borrowed from the disability movement which has been frequently expressed by people with dementia in public campaigns. ${ }^{10}$

To date, knowledge about strategies that promote effective development of dementia-friendly and inclusive communities remains limited, indicating that there is a need to identify enabling strategies to inform education, practice, policy and future research. ${ }^{24}$ In this scoping review study, 'strategy' refers to an action plan and interventions conducted to achieve the goal of creating dementia-friendly and inclusive communities. In June 2019, the Government of Canada released its national dementia strategy. 'Promoting social inclusion and dementia-inclusive communities' was one of the areas of focus in the Canadian dementia strategy. ${ }^{25}$ The Public Health Agency of Canada launched the Dementia Community Investment, a four million dollar per year ongoing investment, which funds community-based projects that seek to achieve the following: optimise the well-being of people with dementia and their informal caregivers; increase public knowledge of dementia and its risk factors; undertake intervention research to assess the effectiveness of the programme or initiative; and apply that knowledge to support expansion of the project's reach to new communities, sectors and populations. ${ }^{11}$

A preliminary search of MEDLINE, CINAHL, PsycINFO and the Joanna Briggs Institute (JBI) Database of Systematic Reviews and Implementation Reports conducted on 30 September 2019 found no systematic review examining strategies that support dementia-friendly and inclusive communities for social inclusion in the community home settings.

In an integrative review, Shannon et alfound eight papers that described the characteristics of dementia-friendly communities. ${ }^{23}$ Building on the work by Shannon et al, our scoping review aims to offer three contributions. First, we will produce a robust synthesis of updated evidence; more studies and reports have emerged in the last 3 years. The articles reported by Shannon et al were published in the year from 2011 to 2016. Second, this scoping review will map accessible literature (including Google search) to 
provide a comprehensive overview of evidence to inform education, practice, policy and further research. Third, by including patient and family partners in conducting the scoping review, we increase the relevance, quality of the study, including transparency and accountability. ${ }^{26}$ As pointed out by the JBI, when a research topic is new and has not been fully established, scoping reviews are useful to systematically map and synthesise the current state of knowledge. ${ }^{27}$

The main objective of the scoping review is to chart the body of literature on strategies and impacts of creating dementia-friendly and inclusive communities that support people with dementia and their informal care providers.

\section{Review questions}

- What social inclusion strategies have been reported in the literature of dementia-friendly and inclusive communities?

- What strategies for developing dementia-friendly and inclusive communities have shown to improve social inclusion?

\section{METHODS}

The proposed scoping review will be conducted in accordance with the JBI methodology for scoping reviews. ${ }^{27}$ This scoping study will take place between April and September 2020.

\section{Inclusion criteria \\ Participants}

This review will include young and older people with dementia living at home in the community. Studies that focus on neighbours, local citizens, public and private service providers, care providers and families of people with dementia in the community that promote dementiafriendly community will also be included.

\section{Concept}

This scoping review aims to identify strategies for developing dementia-friendly and inclusive communities that have shown to improve social inclusion. The core concept is strategy. Such strategies may include public education activities that change attitudes and behaviours to reduce stigma in a community or any interventions that create positive impact to improve social inclusion and social participation of people with dementia. For example, any articles that report on public awareness initiatives, education and training about dementia, development of physical environment guidelines will be considered. 'Impact' refers to a broad range of changes that occur as a result of dementia-friendly and inclusive community activities. We will examine information that reports reach, adoption and implementation. ${ }^{28}$ Improved public knowledge of dementia and increased social participation of people with dementia are examples of positive impact of social inclusion.

\section{Context}

Community in this review refers to people residing at home in a local geographical area. Studies in targeted formal healthcare organisations such as long-term care facilities and hospitals will be not considered in this review.

\section{Types of studies}

This scoping review will consider studies published in English with no time limit. A wide range of study designs from randomised controlled trials to descriptive studies will be considered. All type of study designs (quantitative and qualitative) will be considered for inclusion. This review will also consider student theses published by universities.

\section{Search strategy}

As recommended in JBI review guidelines, we will apply the three-step search strategy. ${ }^{29}$ The first step is an initial limited search of at least two appropriate online databases relevant to the topic. An initial limited search of MEDLINE and CINAHL will be conducted using the selected keywords: dementia or Alzheimer, (community or communities) OR (city or cities) OR (neighbourhood or neighbourhood) OR (environment or environments), friendly or capable or inclusive or inclusion. The initial search will be followed by analysis of the text words contained in the titles and abstracts of relevant articles, as well as the index terms used to describe these articles. The second step involves using all identified keywords and index terms, will then be undertaken and adapted for each included information source. Third, the reference lists of all included articles and reports will be screened for additional studies. A full search strategy for MEDLINE is included in (see online supplementary file 1 for the selected keywords and details of the three-step approach). We have worked and will continue to work with a gerontology librarian at the university to refine the search strategy to ensure key articles are captured. The senior scientist (AP) in the team is familiar with key literature and will provide guidance for specific reference search throughout the process.

\section{Information sources}

The databases to be searched include MEDLINE, CINAHL, Ageline, PsycINFO, Web of Science and ProQuest for thesis and dissertation. Google will be searched as well by using phrases, that is, 'dementia-friendly' OR 'dementia friendly' OR 'dementia-inclusive' OR 'dementia inclusive' OR 'dementia-capable' OR 'dementia capable'.

\section{Study selection}

Following the search, all identified citations will be collated and uploaded into Mendeley and duplicates removed. Titles and abstracts will then be screened by two independent researchers (LH and SL) for assessment against the inclusion criteria for the review. Potentially relevant studies will be retrieved in full, and their citation details imported into the Joanna Briggs Institute System 
for the Unified Management, Assessment and Review of Information (JBI, Adelaide, Australia). The full text of selected citations will be assessed in detail against the inclusion criteria by two independent researchers. The first two authors will check with the gerontology librarian and senior scientist (AP) to refine the searching and selecting process. Reasons for exclusion of full-text studies that do not meet the inclusion criteria will be recorded and reported in the scoping review. Any disagreements that arise among the reviewers at each stage of the study selection process will be resolved through discussion. If consensus cannot be achieved, the senior scientist (AP) will facilitate discussion to foster the decision-making process. The results of the search will be reported in full in the final report and presented in a Preferred Reporting Items for Systematic Reviews and Meta-analyses for Scoping Reviews flow diagram.

\section{Data extraction}

Data will be extracted from papers included in the scoping review by two researchers using a data extraction tool. The data extracted will include specific details about the year of publication, country, setting, population, strategies and impacts related to dementia-friendly and inclusive communities that support social inclusion. For example, in 2019, Phillipson et al reported successful strategies such as education events codesigned and cofacilitated by people with dementia at Kiama in Australia. ${ }^{13}$ The quantitative data in survey (eg, attitude and knowledge change) conducted with community members will be extracted as impact. Data about the number of community members received education activities will also be extracted as project reach under impact. We will also extract relevant qualitative responses, such as experiences and perspectives. A draft charting table is provided (see online supplementary file 2 ). The data extraction tool will be pilot-tested; two independent researchers will complete extraction from three studies and compare results. The draft data extraction tool will be modified and revised as necessary during the process of extracting data from each included study. Modifications will be detailed in the full scoping review report. Based on the discussion in study team meeting, we are open to go back to any included articles to further explore and present results beyond extracted data if needed. Any disagreements that arise between the reviewers will be resolved through discussion. If consensus cannot be reached, the senior scientist (AP) will be consulted.

\section{Patient and public involvement}

Three patient partners (people with early stage of dementia-MG, JM and LJ) and four family partners (people who have a family member with dementia- $\mathrm{NH}, \mathrm{LW}, \mathrm{AB}$ and CW) will be engaged in regular meetings to discuss extracted data and validate study results. See their full names in acknowledgement. Patient and family partners will review extracted data and full text articles. Each patient and family partners will decide the number of articles that they would like to review. We anticipate about 3-5 articles per person on average. Patient and family partners will receive a small honorarium, and they will be coauthors of the next paper that reports the scoping study results. Patient and family partners were recruited from a local community organisation, the Community Engagement Advisory Network (CEAN). More information about how CEAN supports patient and public involvement can be found at http://cean.vch.ca. Also, we will invite organisational leaders in local health authorities and representatives of local Alzheimer Society chapters to engage in a day-long workshop. If in-person meeting is not possible due to the COVID-19 (coronavirus) situation, we will engage stakeholders by zoom, video-conferencing meeting. The aim of the workshop is to discuss input and seek feedback on the scoping review results, with the goal of having these workshop participants disseminate the results on their respective organisational websites and their networks of communication.

\section{Ethics and dissemination}

Research ethics approval and consent to participate is not required for this scoping review. The results will be disseminated at regional, national and international conferences. The findings will be made accessible to health professionals, policy and decision-makers, and the public.

\section{Data synthesis}

The extracted data and results will be presented in table to summarise and map the existing literature. A narrative summary will accompany the tabled results to describe the characteristics of the literature on dementia-friendly and inclusive community strategies that support social inclusion. The categories that will be used for data presentation include: year of publication, country, context, population, intervention types, enabling strategies and impacts related to dementia-friendly and inclusive communities that support social inclusion. The authors anticipate the findings will be a critical step in providing evidence-based guidance to inform future practice, policy and research.

\section{Twitter Lillian Hung @nurselillian}

Acknowledgements The authors wish to thank the Gerontology Research Librarian, Nina Smart at Simon Fraser University for her assistance. We are also grateful for the careful edits of the final manuscript by Annette Berndt, a family partner and a member of the Community Engagement Advisory Network. We also want to thank patient and family partners for their involvement in the work of the scoping review study. Our patient partners are: Mario Gregorio, Jim Mann, Lynn Jackson and our family partners are: Neil Horne, Lily Wong, Annette Berndt and Christine Wallsworth.

Contributors LH conceived the idea, developed the research protocol and methods, and drafted and edited the final manuscript. SL, RH and AP helped to refine and develop the research question and study methods and made meaningful contributions to the drafting and editing of the manuscript. All authors approved the final manuscript submitted.

Funding This review is funded by the Michael Smith Foundation for Health Research \#18 773.

Competing interests None declared. 
Patient and public involvement Patients and/or the public were involved in the design, or conduct, or reporting, or dissemination plans of this research. Refer to the Methods section for further details.

Patient consent for publication Not required.

Provenance and peer review Not commissioned; externally peer reviewed.

Open access This is an open access article distributed in accordance with the Creative Commons Attribution Non Commercial (CC BY-NC 4.0) license, which permits others to distribute, remix, adapt, build upon this work non-commercially, and license their derivative works on different terms, provided the original work is properly cited, appropriate credit is given, any changes made indicated, and the use is non-commercial. See: http://creativecommons.org/licenses/by-nc/4.0/.

ORCID iD

Lillian Hung http://orcid.org/0000-0002-7916-2939

\section{REFERENCES}

1 World Health Organization. Dementia, 2019. Available: https://www. who.int/news-room/fact-sheets/detail/dementia [Accessed 10 Aug 2019].

2 Courtin E, Knapp M, isolation S. Social isolation, loneliness and health in old age: a scoping review. Health Soc Care Community 2017;25:799-812.

3 Dean J, Silversides K, Crampton J. Evaluation of the Bradford dementia friendly communities programme programme. Joseph Rowntree Foundation, 2015.

4 Webster D. Dementia-friendly communities Ontario : a multi-sector collaboration to improve quality of life for people living with dementia and care partners Ontario. Toronto, ON, 2016.

5 Wiersma EC, Denton A. From social network to safety net: dementia-friendly communities in rural Northern Ontario. Dementia 2016;15:51-68.

6 Phinney A, Kelson E, Baumbusch J, et al. Walking in the neighbourhood: performing social citizenship in dementia. Dementia 2016;15:381-94.

7 Phinney A, Chaudhury H, O'Connor DL. Doing as much as I can do: the meaning of activity for people with dementia. Aging Ment Health 2007;11:384-93.

8 Wiersma EC. The experiences of place: veterans with dementia making meaning of their environments. Health Place 2008;14:779-94.

9 Lin S-Y, Lewis FM, friendly D. Dementia friendly, dementia capable, and dementia positive: concepts to prepare for the future. Gerontologist 2015;55:237-44.

10 Wolfe A. Dementia friendly community: municipal toolkit. Saskatchewan, 2017. Available: http://www.dementiafriendlysask atchewan.ca/assets/dfc_municipal_toolkit_web.pdf
11 Ogilvie K, Eggleton A, Standing Senate Committee on Social Affairs, Science and Technology. Dementia in Canada: a national strategy for dementia-friendly communities, 2016. Available: www.senate-senat. ca [Accessed 27 Dec 2019].

12 Hebert CA, Scales K. Dementia friendly initiatives: a state of the science review. Dementia 2019;18:1858-95.

13 Phillipson L, Hall D, Cridland E, et al. Involvement of people with dementia in raising awareness and changing attitudes in a dementia friendly community pilot project. Dementia 2019;18:2679-94.

14 Rahman S, Swaffer K. Assets-based approaches and dementiafriendly communities. Dementia 2018;17:131-7.

15 Woodward M, Arthur A, Darlington N, et al. The place for dementia-friendly communities in England and its relationship with epidemiological need. Int J Geriatr Psychiatry 2019;34:67-71.

16 Alzheimer Society of UK. What is a dementia- friendly community? 2019: 1-5.

17 Phillipson L, Hall D, Cridland E. Dementia friendly Kiama pilot project evaluation. Wollongong, Australia, 2016.

18 Heward M, Innes A, Cutler C, et al. Dementia-friendly communities: challenges and strategies for achieving stakeholder involvement. Health Soc Care Community 2017;25:858-67.

19 Australia A. Living with dementia in the community. Hawker, 2014. Available: https://www.dementia.org.au/sites/default/files/Dementia FriendlySurvey_Final_web.pdf

20 Courtney-Pratt H, Mathison K, Doherty K. Distilling authentic community-led strategies to support people with dementia to live well. Community Dev 2018;49:432-49.

21 Fleming R, Bennett K, Preece T, et al. The development and testing of the dementia friendly communities environment assessment tool (DFC eat). Int Psychogeriatr 2017;29:303-11.

22 Mitchell L, Burton E, Raman S. Dementia-friendly cities: designing intelligible neighbourhoods for life. J Urban Des 2004;9:89-101.

23 Shannon K, Bail K, Neville S. Dementia-friendly community initiatives: an integrative review. J Clin Nurs 2019;28:2035-45.

24 Buckner S, Darlington N, Woodward M, et al. Dementia friendly communities in England: a scoping study. Int J Geriatr Psychiatry 2019;34:1235-43.

25 Alzheimer Society Canada. Canada's national dementia strategy. Available: http://alzheimer.ca/en/Home/Get-involved/Advocacy/ National-dementia-strategy-guide [Accessed 27 Dec 2019].

26 Hung L, Berndt A, Wallsworth C, et al. Involving patients and families in a social robot study. Patient Exp J 2019;6:66-74.

27 Peters M, Godfrey C, Khalil H. The Joanna Briggs institute reviewers' manual, 2015.

28 Sweet SN, Ginis KAM, Estabrooks PA, et al. Operationalizing the REAIM framework to evaluate the impact of multi-sector partnerships. Implement Sci 2014;9:1-10.

29 Peters M, Godfrey-smith P, Mcinerney P, et al. Guidance for the Conduct of JBI Scoping Reviews. In: Aromataris E, Munn Z, eds. Joanna Briggs institute reviewer's manual. South Australia: Joanna Briggs Institute, 2019. https://reviewersmanual.joannabriggs.org/ 